\title{
Quantum critical transport in clean graphene
}

\author{
Lars Fritz, ${ }^{1}$ Jörg Schmalian, ${ }^{2}$ Markus Müller, ${ }^{1}$ and Subir Sachdev ${ }^{1}$ \\ ${ }^{1}$ Department of Physics, Harvard University, Cambridge MA 02138, USA \\ ${ }^{2}$ Ames Laboratory and Department of Physics and Astronomy, \\ Iowa State University, Ames, IA 50011, USA
}

(Dated: October 26, 2018)

\begin{abstract}
We describe electrical transport in ideal single-layer graphene at zero applied bias. There is a crossover from collisionless transport at frequencies larger than $k_{B} T / \hbar$ ( $T$ is the temperature) to collision-dominated transport at lower frequencies. The d.c. conductivity is computed by the solution of a quantum Boltzmann equation. Due to a logarithmic singularity in the collinear scattering amplitude (a consequence of relativistic dispersion in two dimensions) quasi-particles and -holes moving in the same direction tend to an effective equilibrium distribution whose parameters depend on the direction of motion. This property allows us to find the non-equilibrium distribution functions and the quantum critical conductivity exactly to leading order in $1 /|\log (\alpha)|$ where $\alpha$ is the coupling constant characterizing the Coulomb interactions.
\end{abstract}




\section{INTRODUCTION}

Despite the intense experimental and theoretical interest in the electronic properties of graphene [1], there has been relatively little progress in measuring and understanding the role of electron-electron interactions. However, the recent ability to grow ultrahigh mobility, suspended, single layer graphene [2, 3] promises that the situation may well change in the near future.

This paper will examine the role of electron-electron interactions in an infinite sample of single layer graphene without impurities. We will also restrict our attention to the undoped case, so that the chemical potential is at the node of the massless Dirac spectrum. Our results can be extended to include a non-zero chemical potential and a dilute concentration of impurities: this was discussed recently in Ref. 4 for a low-frequency 'hydrodynamic' regime, and additional results will appear in forthcoming work.

The key to understanding electron-electron interactions in clean, undoped graphene is the fact that it is a nearly 'quantum critical' system with marginally irrelevant Coulomb interactions [5, 6, 6, 8, 9]. This implies that the inelastic, electron-electron scattering rate is of order $k_{B} T / \hbar$, where $T$ is the absolute temperature, and there is a crossover from 'hydrodynamic' to 'collisionless' transport as the measurement frequency $(\omega)$ is increased past the scattering rate [10, 11]. These two regimes are captured in the following limiting forms for the frequency dependence of the electrical conductivity, $\sigma$,

$$
\sigma(\omega)=\left\{\begin{array}{cc}
\frac{e^{2}}{h}\left[\frac{\pi}{2}+\mathcal{O}\left(\frac{1}{\ln (\Lambda / \hbar \omega)}\right)\right], \quad \hbar \omega \gg k_{B} T \\
\frac{e^{2}}{h \alpha^{2}(T)}\left[0.760+\mathcal{O}\left(\frac{1}{|\ln (\alpha(T))|}\right)\right], & \hbar \omega \ll k_{B} T \alpha^{2}(T)
\end{array},\right.
$$

where $\alpha(T)$ is a temperature-dependent, dimensionless 'fine-structure constant', which controls the strength of the electron-electron interactions (defined more precisely in Section 【I), and $\Lambda$ is a cutoff energy scale of the order of the electronic bandwidth. The high frequency result above (the 'collisionless' regime) was obtained in Refs. 9, 12. The leading term is the conductivity of 4 species of free massless Dirac fermions. Herbut et al. [12] also obtained the coefficient of the subleading $[\ln (\Lambda / \hbar \omega)]^{-1}$ term. The low frequency result in Eq. (1.1), which is the collision-dominated hydrodynamic regime, is the primary new result of this paper. At asymptotically low temperatures we have (see Eq. (2.4 $)$ )

$$
\alpha(T) \approx \frac{4}{\ln (\Lambda / T)}
$$

the resulting logarithmic increase of $\sigma$ with decreasing $T$ is similar to those of quantum

critical systems in their upper-critical dimension [13], and the inelastic scattering rate of the carriers is of order $\left(k_{B} T / \hbar\right) \alpha^{2}(T)$. 
Related results have been obtained recently by Kashuba [14] in a preprint which appeared while our paper was being completed.

We also note that our results are obtained in the context of a solution of the quantum Boltzmann equation. Going beyond the Boltzmann approximation, and in a system with perfect momentum conservation, we have to consider potentially singular hydrodynamic "long-time tails" in a mode-coupling theory [15], which could modify the low frequency behavior of the conductivity. Such effects are however innocuous here, because the longrange Coulomb interaction suppresses density fluctuations.

\section{RENORMALIZATION GROUP ANALYSIS}

Here, and in the remainder of the paper, we set $\hbar=k_{B}=1$.

We begin introducing the low-energy theory for graphene, and reviewing its renormalization group (RG) properties. The theory is expressed in terms of $N=4$ species of two-component Dirac fermions $\Psi_{a}(a=1 \ldots N)$ and the Euclidean partition function

$$
\begin{aligned}
& \mathcal{Z}= \int \mathcal{D} \Psi_{\alpha} \mathcal{D} A_{\tau} \exp (-\mathcal{S}) \\
& \mathcal{S}=\sum_{a=1}^{N} \int d \mathbf{x} \int d \tau \Psi_{a}^{\dagger}(\mathbf{x}, \tau)\left[\frac{\partial}{\partial \tau}+i e A_{\tau}(\mathbf{x}, \tau)+i v_{F}^{0} \sigma^{x}\left(\frac{\partial}{\partial x}+i \frac{e}{c} A_{x}\right)\right. \\
&\left.\quad+i v_{F}^{0} \sigma^{y}\left(\frac{\partial}{\partial y}+i \frac{e}{c} A_{y}\right)\right] \Psi_{a}(\mathbf{x}, \tau)+\frac{1}{2} \int \frac{d^{2} q}{4 \pi^{2}} \int d \tau \frac{\varepsilon q}{2 \pi}\left|A_{\tau}(\mathbf{q}, \tau)\right|^{2}
\end{aligned}
$$

The functional integral is over fields defined in two spatial dimensions $\mathbf{x}=(x, y)$ and imaginary time $\tau, \sigma^{x, y}$ are Pauli matrices acting on the sublattice space of the honeycomb lattice, and $v_{F}^{0}$ is the bare Fermi velocity. The scalar potential, $A_{\tau}$, mediates the $e^{2} /(\varepsilon|\mathbf{x}|)$ Coulomb interaction between the electrons, where $\varepsilon=\left(\varepsilon_{A}+\varepsilon_{B}\right) / 2$ is the dielectric constant for a graphene sheet confined between two dielectrica with dielectric constants $\varepsilon_{A}$ and $\varepsilon_{B}$, respectively. We have also introduced an non-fluctuating external vector potential $\mathbf{A}=\left(A_{x}, A_{y}\right)$ as a source field: this allows us to extract the electrical current.

The renormalization group properties of $\mathcal{Z}$ have been discussed elsewhere [5, 6, 7, 8, 9]. The fermion field $\Psi_{a}$ undergoes a wavefunction renormalization, the charge $e$ remains unrenormalized, and the velocity $v_{F}$ renormalizes to larger values with decreasing energy scale. For the velocity renormalization, we have the RG equation

$$
\frac{d v_{F}}{d \ell}=f(\alpha) v_{F}
$$

where the running "fine-structure constant" is

$$
\alpha \equiv \frac{e^{2}}{\varepsilon v_{F}},
$$


and the function $f(\alpha)=\alpha / 4$ in the perturbative regime of small $\alpha$. We can re-express these results in terms of the RG equation for the dimensionless coupling $\alpha$

$$
\frac{d \alpha}{d \ell}=-\frac{\alpha^{2}}{4}+\mathcal{O}\left(\alpha^{3}\right)
$$

Notice that $\alpha$ scales to small values at small energies, and this is what facilitates the transport analysis of this paper. It has been shown that $\alpha=0$ is the only fixed point in an analysis which, in the large $N$ limit, also remains valid for large values of $\alpha[5,7]$.

We are only interested here in observables related to the electrical current, and so we will not need the explicit form of the wavefunction renormalization. The current is obtained by taking a functional derivative with respect to $\mathbf{A}$, and this is protected by gauge invariance to have the same form when expressed in terms of either the bare or renormalized quantities [13], which we will use explicitly in (3.11]3.12) below. For two dimensional graphene this implies that the scaling dimension of the conductivity is exactly zero and is unaffected by wavefunction renormalizations. This result can also be obtained explicitly by exploring charge conservation of the system along with the related Ward identity [9] and holds to arbitrary order in perturbation theory.

We are interested here in the collision-dominated transport regime, where the characteristic energy of excitations is $k_{B} T$. We thus use the RG equation to scale down from some high energy cutoff scale, $\Lambda$, to a scale $k_{B} T$. Integrating Eq. (2.3) over this regime, we obtain

$$
\alpha(T)=\frac{\alpha^{0}}{1+\left(\alpha^{0} / 4\right) \ln (\Lambda / T)} \stackrel{T \rightarrow 0}{\sim} \frac{4}{\ln (\Lambda / T)},
$$

where $\alpha^{0}$ is the bare value dependent upon $v_{F}^{0}$. Son [5] has also examined the structure of the RG flow at strong coupling in the large $N$ limit; he finds that there is a significant intermediate energy scale over which

$$
\alpha(T) \sim\left(\frac{T}{\Lambda}\right)^{4 /\left(\pi^{2} N\right)}
$$

Both Eqs. (2.4) and (2.5) predict a slow flow with decreasing temperature towards weak coupling. We can also use $\alpha(T)$ to obtain a $T$-dependent velocity

$$
v_{F}(T)=v_{F}^{0} \frac{\alpha^{0}}{\alpha(T)}=v_{F}^{0}\left[1+\frac{\alpha^{0}}{4} \ln (\Lambda / T)\right] .
$$

We also note that the leading order flow in $\alpha$ in Eq. (2.3) represents an exchangecorrelation effect. Ordinary screening effects are formally higher order, and can be accounted for in the random-phase approximation by the mapping [16, 17]

$$
\alpha(T) \rightarrow \frac{\alpha(T)}{1+N \pi \alpha(T) / 8} .
$$




\section{COLLISION-DOMINATED TRANSPORT}

After initially renormalizing down to a scale $T$ as described in Eq. (III), we can now investigate the transport quantities in the renormalized theory. So all subsequent appearances of the field $\Psi$, the velocity $v_{F}$, and the coupling $\alpha$ implicitly refer to the $T$-dependent renormalized quantities obtained as described in Section II. We will not explicitly write-out this $T$ dependence.

Our formulation of the transport properties of the renormalized theory of weaklyinteracting massless Dirac fermions closely follows that presented in Ref. 11. This previous work considered massless Dirac fermions interacting with a weak statistical interaction due to a Chern-Simons term, and here we only need to replace the Chern-Simons term by a Coulomb interaction. The transport analysis is easiest in the real-time operator formulation with the Hamiltonian

$$
\begin{aligned}
H & =H_{0}+H_{1} \\
H_{0} & =\int d \mathbf{x}\left[v_{F} \Psi_{a}^{\dagger}\left(-i \sigma^{i} \partial_{i}\right) \Psi_{a}\right] \\
H_{1} & =\frac{1}{2} \int \frac{d^{2} k_{1}}{(2 \pi)^{2}} \frac{d^{2} k_{2}}{(2 \pi)^{2}} \frac{d^{2} q}{(2 \pi)^{2}} \Psi_{a}^{\dagger}\left(\mathbf{k}_{2}-\mathbf{q}\right) \Psi_{a}\left(\mathbf{k}_{2}\right) V(\mathbf{q}) \Psi_{b}^{\dagger}\left(\mathbf{k}_{1}+\mathbf{q}\right) \Psi_{b}\left(\mathbf{k}_{1}\right),
\end{aligned}
$$

with the Coulomb interaction

$$
V(\mathbf{q})=\frac{2 \pi e^{2}}{\varepsilon|\mathbf{q}|},
$$

and $a=1, \ldots, N$ labeling the "flavors" of fermions $(N=4$ in graphene, accounting for 2 valleys and 2 spin projections). Even though we compute our results specifically for the Coulomb interactions (3.3), the formalism carries through in exactly the same manner for arbitrary isotropic two body potentials.

The simplest formulation of the transport equations is in a basis which diagonalizes the Hamiltonian $H_{0}$. To do this, we first express $\Psi$ in its Fourier components

$$
\Psi_{a}(\mathbf{x}, t)=\int \frac{d^{2} k}{(2 \pi)^{2}}\left(\begin{array}{c}
c_{1 a}(\mathbf{k}, t) \\
c_{2 a}(\mathbf{k}, t)
\end{array}\right) e^{i \mathbf{k} \cdot \mathbf{x}},
$$

and then perform a unitary transformation from the Fourier mode operators $\left(c_{1 a}, c_{2 a}\right)$ to $\left(\gamma_{+a}, \gamma_{-a}\right)$ :

$$
\begin{aligned}
c_{1 a}(k) & =\frac{1}{\sqrt{2}}\left(\gamma_{+a}(\mathbf{k})+\gamma_{-a}(\mathbf{k})\right) \\
c_{2 a}(k) & =\frac{K}{\sqrt{2} k}\left(\gamma_{+a}(\mathbf{k})-\gamma_{-a}(\mathbf{k})\right) .
\end{aligned}
$$

We have introduced here a notational convention that we shall find quite useful in the following: as $\mathbf{k}$ is a two-dimensional momentum, we can define the complex number $K$ by

$$
K \equiv k_{x}+i k_{y} \quad \text { where } \quad \mathbf{k} \equiv\left(k_{x}, k_{y}\right)
$$


and $k=|\mathbf{k}|=|K|$. Expressing the Hamiltonian $H_{0}$ in terms of $\gamma_{ \pm}$, we obtain the simple result

$$
H_{0}=\sum_{\lambda, a} \int \frac{d^{2} k}{(2 \pi)^{2}} \lambda v_{F} k \gamma_{\lambda a}^{\dagger}(\mathbf{k}) \gamma_{\lambda a}(\mathbf{k})
$$

where the sum over $\lambda$ extends over,+- .

Let us also express the interaction Hamiltonian $H_{1}$ in terms of the $\gamma_{\lambda a}$ :

$$
\begin{aligned}
H_{1}=\sum_{\lambda_{1} \lambda_{2} \lambda_{3} \lambda_{4}} & \int \frac{d^{2} k_{1}}{(2 \pi)^{2}} \frac{d^{2} k_{2}}{(2 \pi)^{2}} \frac{d^{2} q}{(2 \pi)^{2}} \\
& \times T_{\lambda_{1} \lambda_{2} \lambda_{3} \lambda_{4}}\left(\mathbf{k}_{1}, \mathbf{k}_{2}, \mathbf{q}\right) \gamma_{\lambda_{4} b}^{\dagger}\left(\mathbf{k}_{1}+\mathbf{q}\right) \gamma_{\lambda_{3} a}^{\dagger}\left(\mathbf{k}_{2}-\mathbf{q}\right) \gamma_{\lambda_{2} a}\left(\mathbf{k}_{2}\right) \gamma_{\lambda_{1} b}\left(\mathbf{k}_{1}\right)
\end{aligned}
$$

where

$$
T_{\lambda_{1} \lambda_{2} \lambda_{3} \lambda_{4}}\left(\mathbf{k}_{1}, \mathbf{k}_{2}, \mathbf{q}\right)=\frac{V(\mathbf{q})}{8}\left[1+\lambda_{1} \lambda_{4} \frac{\left(K_{1}^{*}+Q^{*}\right) K_{1}}{\left|\mathbf{k}_{1}+\mathbf{q}\right| k_{1}}\right]\left[1+\lambda_{2} \lambda_{3} \frac{\left(K_{2}^{*}-Q^{*}\right) K_{2}}{\left|\mathbf{k}_{2}-\mathbf{q}\right| k_{2}}\right] .
$$

Finally, we also express the electrical current, obtained by taking a functional derivative of the action with respect to $\mathbf{A}$, in terms of the $\gamma_{ \pm}$. For the case of a spatially independent current (which is the only case of interest here), the result can be written as

$$
\mathbf{J}=\mathbf{J}_{I}+\mathbf{J}_{I I}
$$

with

$$
\mathbf{J}_{I}=e v_{F} \sum_{\lambda a} \int \frac{d^{2} k}{(2 \pi)^{2}} \frac{\lambda \mathbf{k}}{k} \gamma_{\lambda a}^{\dagger}(\mathbf{k}) \gamma_{\lambda a}(\mathbf{k})
$$

and

$$
\mathbf{J}_{I I}=-i e v_{F} \int \frac{d^{2} k}{(2 \pi)^{2}} \frac{(\hat{\mathbf{z}} \times \mathbf{k})}{k}\left[\gamma_{+a}^{\dagger}(\mathbf{k}) \gamma_{-a}(\mathbf{k})-\gamma_{-a}^{\dagger}(\mathbf{k}) \gamma_{+a}(\mathbf{k})\right]
$$

where $\hat{\mathbf{z}}$, a unit vector orthogonal to the $x, y$ plane. $\mathbf{J}_{I}$ measures the current carried by motion of the quasiparticles and quasiholes - notice the $\lambda$ prefactor, indicating that these excitations have opposite charges. The operator $\mathbf{J}_{I I}$ creates a quasiparticle-quasihole pair, and in the low frequency limit of interest here we may neglect $\mathbf{J}_{I I}$. Similarly to the problems studied in Refs.[10, 11], a current carying state with holes and electrons moving in opposite directions is consistent with a vanishing total momentum. Thus a finite conductivity does not require the total momentum of the problem to relax. This is the physical reason why at the particle hole symmetric point, i.e., at vanishing deviation of the chemical potential from the Dirac point, the d.c. conductivity is finite even in the absence of momentum relaxing impurities. However, as we will see below at finite deviation from particle hole symmetry a driving electric field always excites the system into a state with finite momentum. The latter cannot decay which entails an infinite d.c. conductivity, in accordance with the hydrodynamic analysis [4]. 
We can now write down the collisionless transport equations for the excitations. As a first step, we define the distribution functions

$$
f_{\lambda}(\mathbf{k}, t)=\left\langle\gamma_{\lambda a}^{\dagger}(\mathbf{k}, t) \gamma_{\lambda a}(\mathbf{k}, t)\right\rangle
$$

where there is no sum over $a$ on the rhs, and we assume the distribution functions to be the same for all valleys and spins. In equilibrium, i.e., in the absence of external perturbations, these are related to the Fermi function

$$
\begin{aligned}
& f_{+}(\mathbf{k}, t)=f^{0}\left(v_{F} k\right)=\frac{1}{e^{\left(v_{F} k-\mu\right) / T}+1} \\
& f_{-}(\mathbf{k}, t)=f^{0}\left(-v_{F} k\right)=\frac{1}{e^{\left(-v_{F} k-\mu\right) / T}+1}
\end{aligned}
$$

where we temporarily allow for a finite chemical potential $\mu$.

Then to first order, in the presence of an external electric field $\mathbf{E}$, we find the simple equations

$$
\left(\frac{\partial}{\partial t}+e \mathbf{E} \cdot \frac{\partial}{\partial \mathbf{k}}\right) f_{\lambda}(\mathbf{k}, t)=0
$$

It is a simple matter to solve (3.15) in linear response. First we parameterize the change in $f_{\lambda}$ from its equilibrium value by [18]

$$
f_{\lambda}(\mathbf{k}, \omega)=2 \pi \delta(\omega) f^{0}\left(\lambda v_{F} k\right)+e \frac{\mathbf{k} \cdot \mathbf{E}(\omega)}{k} f^{0}\left(\lambda v_{F} k\right)\left(1-f^{0}\left(\lambda v_{F} k\right)\right) g_{\lambda}(k, \omega),
$$

where we have performed a Fourier transform in time to frequencies, $\omega$, and introduced the unknown function $g_{\lambda}(k, \omega)$. At the particle hole symmetric point $(\mu=0)$, an applied electric field generates an deviations in the distribution functions having opposite sign for quasiparticles and quasiholes,

$$
g_{\lambda}(k, \omega)=\lambda g(k, \omega) .
$$

This reflects the fact that there is an increased number of quasiholes and quasiparticles moving parallel and antiparallel to field, respectively. As quasiparticles and -holes have opposite charges, their electrical currents are equal, while their net momenta have opposite signs.

Inserting (3.16) into (3.15), we obtain a simple solution for the function $g$

$$
g(k, \omega)=\frac{v_{F} / T}{(-i \omega+\eta)}
$$

where $\eta$ is a positive infinitesimal. Inserting this result into (3.13) and (3.11), we obtain the conductivity

$$
\begin{aligned}
\sigma(\omega)=\frac{\left\langle J_{I}\right\rangle}{E(\omega)} & =2 N \frac{e^{2} v_{F}}{(-i \omega+\eta)} \int \frac{d^{2} k}{(2 \pi)^{2}} \frac{k_{x}^{2}}{k^{2}}\left(-\frac{\partial f^{0}\left(v_{F} k\right)}{\partial k}\right) \\
& =\frac{e^{2}}{h} \frac{N k_{B} T \ln 2}{(-i \hbar \omega+\eta)}
\end{aligned}
$$


where, in the last equation, we have re-inserted factors of $\hbar$ and $k_{B}$. Note that all factors of $v_{F}$ have cancelled out: this is a consequence of the conductivity having scaling dimension $d-2$ (where $d$ is the spatial dimensionality), and being independent of the dynamic critical exponent z. So in this free electron approximation, the real part of the low frequency $\sigma$ is a delta function at $\omega=0$ with weight of order $k_{B} T$.

Including interband transitions the real part of the conductivity becomes

$$
\operatorname{Re} \sigma(\omega)=\frac{e^{2}}{h} N \pi\left[\left[k_{B} T \ln 2\right] \delta(\hbar \omega)+\frac{1}{8} \tanh \left(\frac{\hbar \omega}{4 k_{B} T}\right)\right]
$$

with high frequency limit $\operatorname{Re} \sigma\left(\omega \gg k_{B} T / \hbar\right) \rightarrow e^{2} N \pi /(8 h)$. In the collisionless regime this constant value remains the leading contribution to the conductivity even if one includes the electron-electron Coulomb interaction [9, 12]. Next order corrections are of the form

$$
\operatorname{Re} \sigma\left(\omega \gg k_{B} T / \hbar\right)=\frac{e^{2}}{h} \frac{N \pi}{8}[1+\mathcal{O}(\alpha(\omega))]
$$

where $\alpha(\omega) \approx 4 / \ln (\Lambda / \hbar \omega)$ is the renormalized frequency-dependent 'fine-structure constant' for $k_{B} T \ll \hbar \omega \ll \Lambda$. For $N=4$, this yields the result given in the upper row of Eq. (1.1). Thus, in the collisionless regime, interactions only lead to very small changes of the conductivity. In the following subsections, we will discuss the opposite, collision dominated limit, $\hbar \omega \ll k_{B} T$, and determine how collisions broaden the delta function of Eq. (3.20) to a Drude peak.

\section{A. Quantum Boltzmann equation}

We now include collision terms on the right hand side of (3.15). We can determine these terms by application of Fermi's golden rule [11], or by the explicit derivation presented in the Appendix:

$$
\begin{aligned}
& \left(\frac{\partial}{\partial t}+e \mathbf{E} \cdot \frac{\partial}{\partial \mathbf{k}}\right) f_{\lambda}(\mathbf{k}, t)=-\frac{(2 \pi)}{v_{F}} \int \frac{d^{2} k_{1}}{(2 \pi)^{2}} \frac{d^{2} q}{(2 \pi)^{2}}\{ \\
& \delta\left(k-k_{1}-|\mathbf{k}+\mathbf{q}|+\left|\mathbf{k}_{1}-\mathbf{q}\right|\right) R_{1}\left(\mathbf{k}, \mathbf{k}_{1}, \mathbf{q}\right)\left\{f_{\lambda}(\mathbf{k}, t) f_{-\lambda}\left(\mathbf{k}_{1}, t\right)\left[1-f_{\lambda}(\mathbf{k}+\mathbf{q}, t)\right]\right. \\
& \left.\quad \times\left[1-f_{-\lambda}\left(\mathbf{k}_{1}-\mathbf{q}, t\right)\right]-\left[1-f_{\lambda}(\mathbf{k}, t)\right]\left[1-f_{-\lambda}\left(\mathbf{k}_{1}, t\right)\right] f_{\lambda}(\mathbf{k}+\mathbf{q}, t) f_{-\lambda}\left(\mathbf{k}_{1}-\mathbf{q}, t\right)\right\} \\
& \delta\left(k+k_{1}-|\mathbf{k}+\mathbf{q}|-\left|\mathbf{k}_{1}-\mathbf{q}\right|\right) R_{2}\left(\mathbf{k}, \mathbf{k}_{1}, \mathbf{q}\right)\left\{f_{\lambda}(\mathbf{k}, t) f_{\lambda}\left(\mathbf{k}_{1}, t\right)\left[1-f_{\lambda}(\mathbf{k}+\mathbf{q}, t)\right]\right. \\
& \left.\left.\quad \times\left[1-f_{\lambda}\left(\mathbf{k}_{1}-\mathbf{q}, t\right)\right]-\left[1-f_{\lambda}(\mathbf{k}, t)\right]\left[1-f_{\lambda}\left(\mathbf{k}_{1}, t\right)\right] f_{\lambda}(\mathbf{k}+\mathbf{q}, t) f_{\lambda}\left(\mathbf{k}_{1}-\mathbf{q}, t\right)\right\}\right\}
\end{aligned}
$$


where

$$
\begin{aligned}
& R_{1}\left(\mathbf{k}, \mathbf{k}_{1}, \mathbf{q}\right)=4\left(\left|T_{+--+}\left(\mathbf{k}, \mathbf{k}_{1}, \mathbf{q}\right)-T_{+-+-}\left(\mathbf{k}, \mathbf{k}_{1},-\mathbf{k}-\mathbf{q}+\mathbf{k}_{1}\right)\right|^{2}\right. \\
&\left.+(N-1)\left|T_{+--+}\left(\mathbf{k}, \mathbf{k}_{1}, \mathbf{q}\right)\right|^{2}+(N-1)\left|T_{+-+-}\left(\mathbf{k}, \mathbf{k}_{1},-\mathbf{k}-\mathbf{q}+\mathbf{k}_{1}\right)\right|^{2}\right) \\
& R_{2}\left(\mathbf{k}, \mathbf{k}_{1}, \mathbf{q}\right)=4\left(\frac{1}{2}\left|T_{++++}\left(\mathbf{k}, \mathbf{k}_{1}, \mathbf{q}\right)-T_{++++}\left(\mathbf{k}, \mathbf{k}_{1}, \mathbf{k}_{1}-\mathbf{k}-\mathbf{q}\right)\right|^{2}\right. \\
&\left.+(N-1)\left|T_{++++}\left(\mathbf{k}, \mathbf{k}_{1}, \mathbf{q}\right)\right|^{2}\right)
\end{aligned}
$$

which are illustrated in Fig. 1, The terms proportional to $R_{1}$ represent collisions between oppositely charged particles, while those proportional to $R_{2}$ are collisions between like charges. There are also processes where a particle-hole pair is created: as in Refs. 10, 11, these can be dropped because they have vanishing phase space upon imposition of the energy conservation constraint with dispersion $\varepsilon_{k}=v_{F} k$

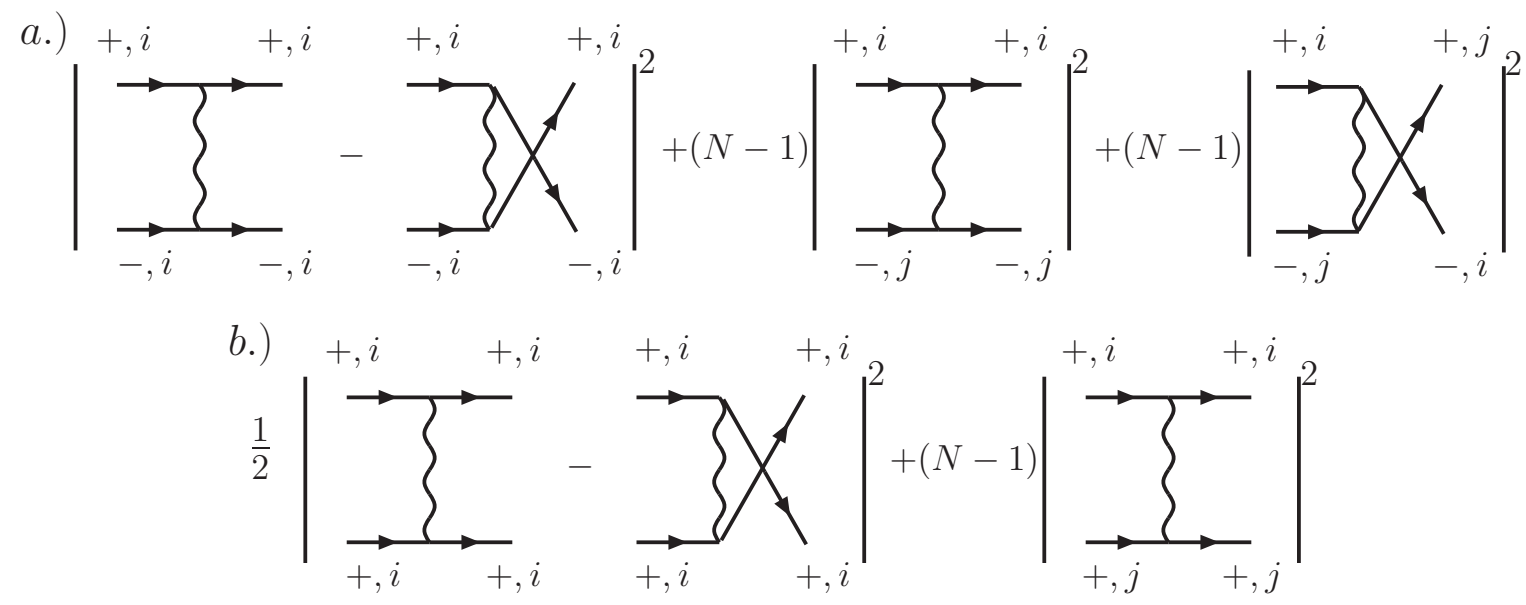

FIG. 1: Illustration of the Golden rule diagrams entering the collision term. The diagrams (a) describe scattering of oppositely charged particles corresponding to the term $R_{1}$, while the diagrams (b) describe scattering of like particles corresponding to the term $R_{2}$. Note that the vertex preserves the flavor $a=i, j$, but not the particle/hole nature $\lambda= \pm$. The factor $1 / 2$ of the first diagram accounts for the symmetry factor associated with having two indistinguishable particles in the final state.

We now proceed to the linearization of (3.23) by inserting the parametrization (3.16) and find 


$$
\begin{aligned}
& \frac{\left(-i \omega g_{\lambda}(k, \omega)-\lambda v_{F} / T\right)}{\left(e^{v_{F} k / T}+1\right)\left(e^{-v_{F} k / T}+1\right)} \frac{\mathbf{k}}{k}=-\frac{(2 \pi)}{v_{F}} \int \frac{d^{2} k_{1}}{(2 \pi)^{2}} \frac{d^{2} q}{(2 \pi)^{2}}\{ \\
& \frac{\delta\left(k-k_{1}-|\mathbf{k}+\mathbf{q}|+\left|\mathbf{k}_{1}-\mathbf{q}\right|\right) R_{1}\left(\mathbf{k}, \mathbf{k}_{1}, \mathbf{q}\right)}{\left(e^{-v_{F} k / T}+1\right)\left(e^{v_{F} k_{1} / T}+1\right)\left(e^{v_{F}|\mathbf{k}+\mathbf{q}| / T}+1\right)\left(e^{-v_{F}\left|\mathbf{k}_{1}-\mathbf{q}\right| / T}+1\right)} \\
& \times\left[\frac{\mathbf{k}}{k} g_{\lambda}(k, \omega)+\frac{\mathbf{k}_{1}}{k_{1}} g_{-\lambda}\left(k_{1}, \omega\right)-\frac{(\mathbf{k}+\mathbf{q})}{|\mathbf{k}+\mathbf{q}|} g_{\lambda}(|\mathbf{k}+\mathbf{q}|, \omega)-\frac{\left(\mathbf{k}_{1}-\mathbf{q}\right)}{\left|\mathbf{k}_{1}-\mathbf{q}\right|} g_{-\lambda}\left(\left|\mathbf{k}_{1}-\mathbf{q}\right|, \omega\right)\right] \\
& +\frac{\delta\left(k+k_{1}-|\mathbf{k}+\mathbf{q}|-\left|\mathbf{k}_{1}-\mathbf{q}\right|\right) R_{2}\left(\mathbf{k}, \mathbf{k}_{1}, \mathbf{q}\right)}{\left(e^{-v_{F} k / T}+1\right)\left(e^{-v_{F} k_{1} / T}+1\right)\left(e^{v_{F}|\mathbf{k}+\mathbf{q}| / T}+1\right)\left(e^{v_{F}\left|\mathbf{k}_{1}-\mathbf{q}\right| / T}+1\right)} \\
& \left.\times\left[\frac{\mathbf{k}}{k} g_{\lambda}(k, \omega)+\frac{\mathbf{k}_{1}}{k_{1}} g_{\lambda}\left(k_{1}, \omega\right)-\frac{(\mathbf{k}+\mathbf{q})}{|\mathbf{k}+\mathbf{q}|} g_{\lambda}(|\mathbf{k}+\mathbf{q}|, \omega)-\frac{\left(\mathbf{k}_{1}-\mathbf{q}\right)}{\left|\mathbf{k}_{1}-\mathbf{q}\right|} g_{\lambda}\left(\left|\mathbf{k}_{1}-\mathbf{q}\right|, \omega\right)\right]\right\} .
\end{aligned}
$$

The remainder of this paper is focused on the solution of the linearized transport equation in Eq. (3.24) for the function $g$. It is useful at this point to recall some crucial mathematical properties of such transport equations, reviewed, e.g., by Ziman [19] and Arnold et al. [18]. We can view the right hand side of Eq. (3.24) as a linear operator, the so-called collision operator $\mathcal{C}$, acting on the function $(\mathbf{k} / k) g(k)$; we drop the implicit $\omega$ dependence because $\mathcal{C}$ is independent of $\omega$. A key property of $\mathcal{C}$ is that it is Hermitian with respect to the natural inner product

$$
\left\langle g_{1} \mid g_{2}\right\rangle \equiv \sum_{\lambda} \int \frac{d^{2} k}{(2 \pi)^{2}} g_{1, \lambda}(k) g_{2, \lambda}(k) .
$$

This Hermiticity follows [18] from symmetry properties of $R_{1}$ and $R_{2}$ under exchanges between incoming and outgoing momenta, which are very similar to those used in establishing Boltzmann's H-theorem.

Related to the above properties of the collision operator, we can introduce a functional $\mathcal{Q}[g]$, such that Eq. (3.24) is equivalent to finding its stationary point

$$
\frac{\delta \mathcal{Q}[g]}{\delta g}=0
$$

Specializing to the particle-hole symmetric case, cf. Eq. (3.17), the explicit form of the 
functional is

$$
\begin{aligned}
& \mathcal{Q}[g]=\frac{(2 \pi)}{8 v_{F}} \int \frac{d^{2} k}{(2 \pi)^{2}} \frac{d^{2} k_{1}}{(2 \pi)^{2}} \frac{d^{2} q}{(2 \pi)^{2}}\{ \\
& \frac{\delta\left(k-k_{1}-|\mathbf{k}+\mathbf{q}|+\left|\mathbf{k}_{1}-\mathbf{q}\right|\right) R_{1}\left(\mathbf{k}, \mathbf{k}_{1}, \mathbf{q}\right)}{\left(e^{-v_{F} k / T}+1\right)\left(e^{v_{F} k_{1} / T}+1\right)\left(e^{v_{F}|\mathbf{k}+\mathbf{q}| / T}+1\right)\left(e^{-v_{F}\left|\mathbf{k}_{1}-\mathbf{q}\right| / T}+1\right)} \\
& \times\left[\frac{\mathbf{k}}{k} g(k, \omega)-\frac{\mathbf{k}_{1}}{k_{1}} g\left(k_{1}, \omega\right)-\frac{(\mathbf{k}+\mathbf{q})}{|\mathbf{k}+\mathbf{q}|} g(|\mathbf{k}+\mathbf{q}|, \omega)+\frac{\left(\mathbf{k}_{1}-\mathbf{q}\right)}{\left|\mathbf{k}_{1}-\mathbf{q}\right|} g\left(\left|\mathbf{k}_{1}-\mathbf{q}\right|, \omega\right)\right]^{2} \\
& +\frac{\delta\left(k+k_{1}-|\mathbf{k}+\mathbf{q}|-\left|\mathbf{k}_{1}-\mathbf{q}\right|\right) R_{2}\left(\mathbf{k}, \mathbf{k}_{1}, \mathbf{q}\right)}{\left(e^{-v_{F} k / T}+1\right)\left(e^{-v_{F} k_{1} / T}+1\right)\left(e^{v_{F}|\mathbf{k}+\mathbf{q}| / T}+1\right)\left(e^{v_{F}\left|\mathbf{k}_{1}-\mathbf{q}\right| / T}+1\right)} \\
& \left.\times\left[\frac{\mathbf{k}}{k} g(k, \omega)+\frac{\mathbf{k}_{1}}{k_{1}} g\left(k_{1}, \omega\right)-\frac{(\mathbf{k}+\mathbf{q})}{|\mathbf{k}+\mathbf{q}|} g(|\mathbf{k}+\mathbf{q}|, \omega)-\frac{\left(\mathbf{k}_{1}-\mathbf{q}\right)}{\left|\mathbf{k}_{1}-\mathbf{q}\right|} g\left(\left|\mathbf{k}_{1}-\mathbf{q}\right|, \omega\right)\right]^{2}\right\} \\
& +\int \frac{d^{2} k}{(2 \pi)^{2}} \frac{g(k, \omega)\left[-i \omega g(k, \omega) / 2-v_{F} / T\right]}{\left(e^{v_{F} k / T}+1\right)\left(e^{-v_{F} k / T}+1\right)} .
\end{aligned}
$$

\section{B. Translational invariance and momentum conservation}

The translational invariance of the system immediately implies the presence of a zero mode of the operator $\mathcal{C}$, which corresponds to the shift of the distribution functions arising from changing to a linearly moving reference frame. The corresponding deviation $g_{\lambda}(k, \omega)$ has the form

$$
g_{\lambda}(k, \omega)=C(\omega) \psi_{t r}(k) \equiv C(\omega) k,
$$

which is easily seen to annihilate the left hand side of (3.24) due to momentum conservation. Note that this zero mode of the Boltzmann operator is orthogonal to any modes of the form (3.17) which are the only ones that can be excited by an electric field at particle hole

symmetry. This again expresses the fact that current and momentum are independent of each other at this special point. However, away from the Dirac point, or if a thermal gradient is applied instead of an electric field, the zero mode $\psi_{t r}$ will be excited by the driving field which leads to a diverging d.c. response in clean systems. This will be discussed in more detail in a forthcoming publication.

For the following we restrict to electrical conductivity at the particle-hole symmetric point where the above zero mode is irrelevant.

\section{Collinear limit}

In the previous analysis of a quantum Boltzmann equation for massless Dirac fermions in two dimensions [11], it was noted that the phase space for scattering of particles was logarithmically divergent in the collinear limit. For the interaction considered in that paper, 
the collinear scattering cross-section vanished, and so this singular phase space density had no important consequences. The collinear scattering does not vanish for the present Coulomb interaction, and so we need to consider this logarithmic divergence seriously.

The physical origin of the divergent collinear scattering is related to the linear dispersion which implies that quasiparticles or -holes moving in the same direction share the same group velocity, independent of their energies. This leads to a diverging duration of collisions of nearly collinear particles, which is enhanced due to the low space dimensionality. To the extent that collinear scattering is very strong, and if we consider small enough frequencies, we may expect that quasiparticles and holes that move in the same direction in the plane will establish a pseudo-equilibrium characterized by an effective chemical potential and an effective temperature which will however depend on the direction of motion.

In linear response the deviations of these effective parameters from the equilibrium values $\mu$ and $T$ have to vary with $\mathbf{k} / k \cdot \mathbf{E}$ for symmetry reasons. Further, the effective temperature shift is easily shown to be identical to the mode $\psi_{t r}$ discussed above, and thus, it is thus ruled out at the particle hole symmetric point $\mu=0$. The remaining dominant mode of the function $g$ will correspond to an effective shift in chemical potential which translates into

$$
g_{\lambda}(k, \omega)=C(\omega) \psi_{\mu}(k) \equiv \frac{v_{F}}{T^{2}} \lambda C(\omega),
$$

where the prefactor has been chosen so as to make $C(\omega)$ dimensionless. With this Ansatz, which will be confirmed below, it simply remains to determine the prefactor $C(\omega)$, yielding the leading term in the non-equilibrium distribution. Note that the effective chemical potential shift ranges between $\pm C \hbar v_{F} e E / T$ depending on the direction. Comparing this to the temperature allows us to estimate the threshold electric field strength, $e E_{\text {lin }}=T^{2} / \hbar v_{F}$, below which non-linear effects should remain small.

Let us now review in more detail, how the above physical picture arises in the formalism of the Boltzmann equation. The occurrence of a logarithmic divergence can be seen by allowing the incoming and outgoing momenta to be nearly collinear. Without loss of generality, we choose $\mathbf{k}=(k, 0)$ with $k>0$. Also, we write $\mathbf{k}_{\mathbf{1}}=\left(k_{1}, k_{\perp}\right), \mathbf{q}=\left(q, q_{\perp}\right)$ with $k_{\perp}$ and $q_{\perp}$ small. The divergence in the phase space density of the collision term proportional to $R_{2}$ occurs when $k_{1}>0, k+q>0$ and $k_{1}-q>0$. Likewise, for scattering of oppositely charged particles a divergence occurs when their $k$ vectors are anticollinear which ensures collinear group velocities since $\mathbf{v}_{\mathbf{k}}=\lambda v_{F} \mathbf{k} / k$. In this regime, the argument of the energy conservation delta function of the particle-particle scattering term can be written as

$$
\begin{aligned}
k+k_{1}-|\mathbf{k}+\mathbf{q}|-\left|\mathbf{k}_{1}-\mathbf{q}\right| & \approx \frac{k_{\perp}^{2}}{2 k_{1}}-\frac{q_{\perp}^{2}}{2(k+q)}-\frac{\left(k_{\perp}-q_{\perp}\right)^{2}}{2\left(k_{1}-q\right)} \\
& \equiv-\frac{\left(k+k_{1}\right)}{2(k+q)\left(k_{1}-q\right)}\left(q_{\perp}-\zeta_{1} k_{\perp}\right)\left(q_{\perp}-\zeta_{2} k_{\perp}\right),
\end{aligned}
$$

where $\zeta_{1,2}$ depend upon $k, k_{1}$, and $q$, and are the roots of a quadratic equation which are defined by the expressions above. Then, the phase space density for the $R_{2}$ term is 
proportional to

$$
\begin{gathered}
\int d k_{\perp} d q_{\perp} \delta\left(k+k_{1}-|\mathbf{k}+\mathbf{q}|-\left|\mathbf{k}_{1}-\mathbf{q}\right|\right)=\int \frac{d k_{\perp}}{\left|k_{\perp}\right|} \frac{4(k+q)\left(k_{1}-q\right)}{\left(k+k_{1}\right)\left|\zeta_{1}-\zeta_{2}\right|} \\
=2 \sqrt{\frac{k_{1}(k+q)\left(k_{1}-q\right)}{k}} \int \frac{d k_{\perp}}{\left|k_{\perp}\right|} .
\end{gathered}
$$

The logarithmic divergence as $k_{\perp} \rightarrow 0$ is now evident. This divergence is clearly a consequence of the linear dispersion of the fermions, and the above analysis also makes it clear that it is special to two dimensions. As discussed in Ref. 18 for a similar divergence in a different problem, we expect that this divergence is cutoff by higher-order self energy corrections to the fermions. Such self-energy corrections appear at order $\alpha$ in the perturbation theory, and so the important range of the $k_{\perp}$ integral is between $T / v_{F}$ and $T \alpha / v_{F}$. So we may approximate [18]

$$
\int \frac{d k_{\perp}}{\left|k_{\perp}\right|} \approx 2 \ln (1 / \alpha),
$$

and set $k_{\perp}=q_{\perp}=0$ elsewhere to obtain the leading contribution to the collision integral in the limit $\alpha \rightarrow 0$. Proceeding in this manner, the part of $\mathcal{C}$ on the right-hand side of Eq. (3.24) proportional to $R_{2}$, which we denote $\mathcal{C}_{2}$, becomes

$$
\begin{aligned}
\mathcal{C}_{2}[g] \approx-\frac{\ln (1 / \alpha)}{2 \pi^{3} v_{F}} \frac{\mathbf{k}}{k} \int_{0}^{\infty} d k_{1} \int_{-k}^{k_{1}} \frac{d q}{q^{2}} \sqrt{\frac{k_{1}(k+q)\left(k_{1}-q\right)}{k}} \\
\times \frac{R_{2}\left[g(k, \omega)+g\left(k_{1}, \omega\right)-g(k+q, \omega)-g\left(k_{1}-q, \omega\right)\right]}{\left(e^{-v_{F} k / T}+1\right)\left(e^{-v_{F} k_{1} / T}+1\right)\left(e^{v_{F}(k+q) / T}+1\right)\left(e^{v_{F}\left(k_{1}-q\right) / T}+1\right)} .
\end{aligned}
$$

Consonant with our discussion earlier in this subsection, a key property of the above expression for $\mathcal{C}_{2}$ was noted by Kashuba [14]: the function $g=$ constant is an eigenvector of $\mathcal{C}_{2}$ with zero eigenvalue. The same is also easily seen to apply to the portion $\mathcal{C}_{1}$ of $\mathcal{C}$ which is proportional to $R_{1}$. Indeed, this is just the direction-specific chemical potential shift in Eq. (3.29), which naturally is a zero mode for collinear scattering, since it maintains a pseudo-equilibrium among particles moving in the same direction.

Going beyond the collinear limit, we conclude that there is an eigenvalue of $\mathcal{C}$ which is not proportional to $\ln (1 / \alpha)$ in the limit of small $\alpha$; the corresponding eigenvector is given by a constant $g(k)$ up to corrections of order $[\ln (1 / \alpha)]^{-1}$.

The solution of the Boltzmann equation in Eq. (3.24) requires that we obtain the operator $\mathcal{C}^{-1}$, and the results above allow us to constrain its form in the limit $\ln (1 / \alpha) \gg 1$. Let $|\mu\rangle$ be the eigenvectors of $\mathcal{C}$ with eigenvalues $\lambda_{\mu}$. Then

$$
\mathcal{C}^{-1}=\sum_{\mu} \frac{|\mu\rangle\langle\mu|}{\lambda_{\mu}},
$$

and in the limit of large $\ln (1 / \alpha), \mathcal{C}^{-1}$ is dominated [14] by the eigenvector whose eigenvalue is not proportional to $\ln (1 / \alpha)$. Note that it is quite remarkable that in this limit we can solve the Boltzmann equation essentially exactly. 


\section{Results}

From the reasoning in the previous subsection, we conclude that up to corrections of order $[\ln (1 / \alpha)]^{-1}$, we can choose $g$ to be of the form

$$
g(k, \omega) \approx \frac{v_{F}}{T^{2}} C(\omega)
$$

We insert this parameterization into the functional $\mathcal{Q}[g]$ in Eq. (3.27); the solution of the stationarity condition in Eq. (3.26) is then equivalent to requiring the vanishing of the derivative with respect to $C$. We numerically evaluated the integrals in Eq. (3.27) using an elliptic co-ordinate system to solve the energy conservation constraint [11], and obtained

$$
\mathcal{Q}[g]=\frac{1}{T} \frac{\ln 2}{4 \pi}\left[\kappa \alpha^{2} C^{2}(\omega)-2 C(\omega)-i\left(\frac{\omega}{T}\right) C^{2}(\omega)\right],
$$

with $\kappa=3.646$ for the physical case $N=4$. From the stationarity condition we then obtain

$$
C(\omega)=\frac{1}{-i(\omega / T)+\kappa \alpha^{2}}
$$

The conductivity can be obtained from $C(\omega)$ by combining Eqs. (3.11), (3.16) and (3.35):

$$
\sigma(\omega)=\frac{e^{2}}{h} \frac{N k_{B} T \ln 2}{-i \hbar \omega+\kappa k_{B} T \alpha^{2}},
$$

where we have re-inserted factors of $\hbar$ and $k_{B}$. Notice that the conductivity depends only upon $\alpha(T)$, while all other factors of $v_{F}(T)$ cancel. Notice also the connection to the free particle result in Eq. (3.19) - the only difference is that the infinitesimal $\eta$ has been replaced by the inelastic relaxation energy $\kappa k_{B} T \alpha^{2}$.

\section{CONCLUSIONS}

We conclude by briefly noting the conditions under which our main results for conductivity in Eqs. (3.37), (2.4), and (2.5) may be observed in transport measurements. The key requirement is that $k_{B} T \alpha^{2}$ be the largest infrared energy scale which quenches the ideal Dirac fermion behavior. Thus, the sample size should be larger than the inelastic scattering length $\ell_{\mathrm{ee}} \approx \hbar v_{F} /\left(k_{B} T \alpha^{2}\right)$. Similarly, the elastic mean-free path from impurity scattering should be larger than $\ell_{\mathrm{ee}}$, too. Finally, particle-hole symmetry is also required, and so the bias voltage should be smaller than $k_{B} T \alpha^{2}$.

It is possible to extend our analysis to include all the additional perturbations noted in the previous paragraph, with a treatment of disorder effects following that of Ref. 20. When these perturbations are weak (compared to $k_{B} T \alpha^{2}$ ), then in the collision-dominated regime, a general hydrodynamic analysis is possible: this was presented recently in Ref. 4. Also, in this regime the analysis of the Boltzmann equation greatly simplifies if the interactions 
are weak enough to ensure a strong logarithmic divergence in the collinear channel. The latter establishes pseudoequilibrium along different directions if the inelastic scattering time remains the shortest relevant time scale in the problem. Otherwise, a full analysis of the modified quantum Boltzmann equation is required. These aspects will be discussed in future work.

\section{Acknowledgments}

We thank S. Das Sarma, F. Guinea, P. Kovtun, D. E. Sheehy, and O. Vafek for useful discussions. This research was supported by Deutsche Forschungsgemeinschaft under grant FR 2627/1-1 (LF); by the Swiss National Fund for Scientific Research under grant PA002113151 (MM); by the NSF under grant DMR-0537077 (SS), and by the Ames Laboratory, operated for the U.S. Department of Energy by Iowa State University under Contract No. DE-AC02-07CH11358 (JS).

\section{APPENDIX A: DERIVATION OF THE QUANTUM KINETIC EQUATION}

An alternative derivation of the quantum kinetic equation can be carried out in the framework of closed time contour ordered perturbation theory, as explicited in Chapter 9 of Ref. [21]. The problem we consider falls into the generic class of a system describing particles interacting via a distance-dependent density-density interaction. Thus the Hamiltonian is of the form

$$
H=H_{0}+\frac{1}{2} \int d^{2} \mathbf{r} d^{2} \mathbf{r}^{\prime} V\left(\mathbf{r}, \mathbf{r}^{\prime}\right) \rho(\mathbf{r}) \rho\left(\mathbf{r}^{\prime}\right),
$$

where $\rho(\mathbf{r})$ denotes the particle density at spatial point $\mathbf{r}$. The following considerations are completely generic and apply to any system which falls into the class of Hamiltonians presented in Eq. (A1). The starting point of our discussion is given by Eq.(9-7a) of Ref. [21], which has to be generalized to incorporate a possible matrix structure of the Green's function (in our case the Green's function lives in spinor space within a structure due to the $\mathrm{N}$ spin and valley species, and thus has a $2 N \times 2 N$ structure)

$$
\begin{aligned}
{\left[\partial_{T}-\nabla_{\mathbf{R}} U(\mathbf{R}, T) \nabla_{\mathbf{k}}\right] \mathbf{G}^{<}(\mathbf{k}, \omega ; \mathbf{R}, T)=- } & \mathbf{G}^{<}(\mathbf{k}, \omega ; \mathbf{R}, T) \boldsymbol{\Sigma}^{>}(\mathbf{k}, \omega ; \mathbf{R}, T) \\
+ & \mathbf{G}^{>}(\mathbf{k}, \omega ; \mathbf{R}, T) \boldsymbol{\Sigma}^{<}(\mathbf{k}, \omega ; \mathbf{R}, T)
\end{aligned}
$$

where

$$
\begin{aligned}
\Sigma_{\alpha \beta}^{>,<}(\mathbf{r}, t ; \mathbf{R}, T) \approx & -i^{2} \int d \overline{\mathbf{R}} d \overline{\mathbf{r}} V(\mathbf{R}+\mathbf{r} / 2-\overline{\mathbf{R}}-\overline{\mathbf{r}} / 2) V(\mathbf{R}-\mathbf{r} / 2-\overline{\mathbf{R}}+\overline{\mathbf{r}} / 2) \times \\
\times & \mathbf{G}_{\gamma \delta}^{<,>}(-\overline{\mathbf{r}},-t ; R, T)\left[\mathbf{G}_{\alpha \beta}^{>,<}(\mathbf{r}, t ; R, T) \mathbf{G}_{\delta \gamma}^{>,<}(\overline{\mathbf{r}}, t ; R, T)\right. \\
& \left.-\mathbf{G}_{\alpha \gamma}^{><}(\mathbf{R}+\overline{\mathbf{r}} / 2-\overline{\mathbf{R}}+\mathbf{r} / 2, t ; R, T) \mathbf{G}_{\delta \dot{\gamma}}^{>,<}(\mathbf{R}+\overline{\mathbf{r}} / 2-\overline{\mathbf{R}}+\mathbf{r} / 2, t ; R,(\mathbb{A}))^{3}\right)
\end{aligned}
$$


in the Born approximation (note: double indices are summed over). The corresponding self-energy diagrams are the RPA-type contribution and the maximally crossed diagram, see also Ref. [21]. In a next step, following the treatment in Kadanoff and Baym, we find that the Fourier transform with respect to the relative coordinates (which corresponds to the mixed Wigner transform) of Eq. (A3) reads (note that in the following we drop the dependence on the centre of mass coordinate $\mathbf{R}$ )

$$
\begin{aligned}
\boldsymbol{\Sigma}_{\alpha \beta}^{>,<}(\mathbf{k}, \omega ; T) & =\int \frac{d^{2} \mathbf{k}_{\mathbf{1}}}{(2 \pi)^{2}} \frac{d \omega_{1}}{2 \pi} \frac{d^{2} \mathbf{k}_{\mathbf{2}}}{(2 \pi)^{2}} \frac{d \omega_{2}}{2 \pi} \frac{d^{2} \mathbf{k}_{\mathbf{3}}}{(2 \pi)^{2}} \frac{d \omega_{3}}{2 \pi}(2 \pi)^{3} \delta\left(\mathbf{k}+\mathbf{k}_{\mathbf{1}}-\mathbf{k}_{\mathbf{2}}-\mathbf{k}_{\mathbf{3}}\right) \times \\
& \times \delta\left(\omega+\omega_{1}-\omega_{2}-\omega_{3}\right) \times \\
& \times\left[V\left(\mathbf{k}-\mathbf{k}_{\mathbf{2}}\right) V\left(\mathbf{k}-\mathbf{k}_{\mathbf{2}}\right) \mathbf{G}_{\gamma \delta}^{<,>}\left(\mathbf{k}_{\mathbf{1}}, \omega_{1}\right) \mathbf{G}_{\alpha \beta}^{>,<}\left(\mathbf{k}_{\mathbf{2}}, \omega_{2}\right) \mathbf{G}_{\delta \gamma}^{>,<}\left(\mathbf{k}_{\mathbf{3}}, \omega_{3}\right)\right. \\
& \left.-V\left(\mathbf{k}-\mathbf{k}_{\mathbf{2}}\right) V\left(\mathbf{k}-\mathbf{k}_{\mathbf{3}}\right) \mathbf{G}_{\gamma \delta}^{<,>}\left(\mathbf{k}, \omega_{1}\right) \mathbf{G}_{\alpha \gamma}^{>,<}\left(\mathbf{k}_{\mathbf{2}}, \omega_{2}\right) \mathbf{G}_{\delta \beta}^{>,<}\left(\mathbf{k}_{\mathbf{3}}, \omega_{3}\right)\right] .
\end{aligned}
$$

Until now all the formulae are completely generic and not specific to graphene. In order to make connection to the problem of graphene we note that the Green's function of the spinors $\Psi$ is related to the Green's function of the $\gamma$ through

$$
\mathbf{G}^{<,>}(\mathbf{k}, \omega)=U_{\mathbf{k}}^{-1} g^{<,>}(\mathbf{k}, \omega) U_{\mathbf{k}}
$$

where the unitary matrix $U_{\mathbf{k}}^{-1}$ according to Eq. (3.5) is given by

$$
U_{\mathbf{k}}^{-1}=\frac{1}{\sqrt{2} k}\left(\begin{array}{cc}
k & k \\
K & -K
\end{array}\right) .
$$

Furthermore we note, that the summation over spin and valley indices only affects the RPAlike diagram, which thus receives a prefactor $N$ and the resulting matrix equation is an equation, whose indices only carry over the $2 \times 2$ spinor. This allows to rewrite Eq. (A4) as

$$
\begin{aligned}
\Sigma_{\alpha \beta}^{>,<}(\mathbf{k}, \omega ; T) & =\int \frac{d^{2} \mathbf{k}_{\mathbf{1}}}{(2 \pi)^{2}} \frac{d \omega_{1}}{2 \pi} \frac{d^{2} \mathbf{k}_{\mathbf{2}}}{(2 \pi)^{2}} \frac{d \omega_{2}}{2 \pi} \frac{d^{2} \mathbf{k}_{\mathbf{3}}}{(2 \pi)^{2}} \frac{d \omega_{3}}{2 \pi}(2 \pi)^{3} \delta\left(\mathbf{k}+\mathbf{k}_{\mathbf{1}}-\mathbf{k}_{\mathbf{2}}-\mathbf{k}_{\mathbf{3}}\right) \times \\
& \times \delta\left(\omega+\omega_{1}-\omega_{2}-\omega_{3}\right) \times \\
& \times\left[N V\left(\mathbf{k}-\mathbf{k}_{\mathbf{2}}\right) V\left(\mathbf{k}-\mathbf{k}_{\mathbf{2}}\right) \times\right. \\
& \times\left(U_{\mathbf{k}_{\mathbf{1}}}^{-1} g^{<,>}\left(\mathbf{k}_{\mathbf{1}}, \omega_{1}\right) U_{\mathbf{k}_{\mathbf{1}}}\right)_{\gamma \delta}\left(U_{\mathbf{k}_{\mathbf{2}}}^{-1} g^{>,<}\left(\mathbf{k}_{\mathbf{2}}, \omega_{2}\right) U_{\mathbf{k}_{\mathbf{2}}}\right)_{\alpha \beta}\left(U_{\mathbf{k}_{3}}^{-1} g^{>,<}\left(\mathbf{k}_{\mathbf{3}}, \omega_{3}\right) U_{\mathbf{k}_{3}}\right)_{\delta \gamma} \\
& -V\left(\mathbf{k}-\mathbf{k}_{\mathbf{2}}\right) V\left(\mathbf{k}-\mathbf{k}_{\mathbf{3}}\right) \\
& \left.\left.\times\left(U_{\mathbf{k}_{\mathbf{1}}}^{-1} g^{<,>}\left(\mathbf{k}_{\mathbf{1}}, \omega_{1}\right) U_{\mathbf{k}_{\mathbf{1}}}\right)_{\gamma \delta}\left(U_{\mathbf{k}_{\mathbf{2}}}^{-1} g^{>,<}\left(\mathbf{k}_{\mathbf{2}}, \omega_{2}\right) U_{\mathbf{k}_{\mathbf{2}}}\right)_{\alpha \gamma}\left(U_{\mathbf{k}_{3}}^{-1} g^{>,<}\left(\mathbf{k}_{\mathbf{3}}, \omega_{3}\right) U_{\mathbf{k}_{\mathbf{3}}}\right\rangle_{\delta \beta} A_{j}\right]\right)
\end{aligned}
$$

Accounting for the fact that the operators $\gamma$ describe sharp quasiparticles the lesser and greater Green's functions are given by

$$
g_{\lambda \lambda^{\prime}}^{<}(\mathbf{k}, \omega ; T)=2 \pi \delta\left(\omega-\epsilon_{\lambda}(\mathbf{k}, T)\right) f_{\lambda}(\mathbf{k}, T) \delta_{\lambda, \lambda^{\prime}}
$$

and

$$
g_{\lambda \lambda^{\prime}}^{>}(\mathbf{k}, \omega ; T)=2 \pi \delta\left(\omega-\epsilon_{\lambda}(\mathbf{k}, T)\right)\left[1-f_{\lambda}(\mathbf{k}, T)\right] \delta_{\lambda, \lambda^{\prime}},
$$


where we assumed the distribution function of the quasiparticles to have no off-diagonal components, which is justified to linear order in the potential gradient. We can formulate the kinetic equation for the diagonal part of the distribution function as

$$
\begin{aligned}
{\left[\partial_{T}-\nabla_{\mathbf{R}} U(\mathbf{R}, T) \nabla_{\mathbf{k}}\right] f_{\mu}(\mathbf{k}, T)=- } & f_{\mu}(\mathbf{k}, T)\left(U_{\mathbf{k}} \boldsymbol{\Sigma}^{>}\left(\mathbf{k}, \omega=\epsilon_{\mu}(\mathbf{k}) ; T\right) U_{\mathbf{k}}^{-1}\right)_{\mu \mu} \\
+ & {\left[1-f_{\mu}(\mathbf{k}, T)\right]\left(U_{\mathbf{k}} \boldsymbol{\Sigma}^{<}\left(\mathbf{k}, \omega=\epsilon_{\mu}(\mathbf{k}) ; T\right) U_{\mathbf{k}}^{-1}\right)_{\mu \mu} }
\end{aligned}
$$

or equivalently

$$
\begin{aligned}
{\left[\partial_{T}-\nabla_{\mathbf{R}} U(\mathbf{R}, T) \nabla_{\mathbf{k}}\right] f_{\mu}(\mathbf{k}, T)=- } & f_{\mu}(\mathbf{k}, T) \sigma_{\mu \mu}^{>}\left(\mathbf{k}, \omega=\epsilon_{\mu}(\mathbf{k}) ; T\right) \\
+ & {\left[1-f_{\mu}(\mathbf{k}, T)\right] \sigma_{\mu \mu}^{<}\left(\mathbf{k}, \omega=\epsilon_{\mu}(\mathbf{k}) ; T\right) . }
\end{aligned}
$$

Exploiting the form of the lesser and greater Green's functions we can rewrite the selfenergies as (note that $\mu$, in contrast to the other double indices, is not summed over here and subsequently)

$$
\begin{aligned}
& \sigma_{\mu \mu}^{>}\left(\mathbf{k}, \omega=\epsilon_{\mu}(\mathbf{k}) ; T\right)=\int \frac{d^{2} \mathbf{k}_{\mathbf{1}}}{(2 \pi)^{2}} \frac{d \omega_{1}}{2 \pi} \frac{d^{2} \mathbf{k}_{\mathbf{2}}}{(2 \pi)^{2}} \frac{d \omega_{2}}{2 \pi} \frac{d^{2} \mathbf{k}_{\mathbf{3}}}{(2 \pi)^{2}} \frac{d \omega_{3}}{2 \pi}(2 \pi)^{3} \delta\left(\mathbf{k}+\mathbf{k}_{\mathbf{1}}-\mathbf{k}_{\mathbf{2}}-\mathbf{k}_{\mathbf{3}}\right) \\
\times & \delta\left(\epsilon_{\mu}(\mathbf{k})+\omega_{1}-\omega_{2}-\omega_{3}\right) \times \\
\times & {\left[N V\left(\mathbf{k}-\mathbf{k}_{\mathbf{2}}\right) V\left(\mathbf{k}-\mathbf{k}_{\mathbf{2}}\right)(2 \pi)^{3} \delta\left(\omega_{1}-\epsilon_{\lambda}\left(\mathbf{k}_{\mathbf{1}}\right)\right) \delta\left(\omega_{2}-\epsilon_{\lambda_{1}}\left(\mathbf{k}_{\mathbf{2}}\right)\right) \delta\left(\omega_{3}-\epsilon_{\lambda_{2}}\left(\mathbf{k}_{\mathbf{3}}\right)\right)\right.} \\
\times & M_{\lambda_{2} \lambda}\left(\mathbf{k}_{\mathbf{3}}, \mathbf{k}_{\mathbf{1}}\right) M_{\lambda \lambda_{2}}\left(\mathbf{k}_{\mathbf{1}}, \mathbf{k}_{\mathbf{3}}\right) M_{\mu \lambda_{1}}\left(\mathbf{k}, \mathbf{k}_{\mathbf{2}}\right) M_{\lambda_{1} \mu}\left(\mathbf{k}_{\mathbf{2}}, \mathbf{k}\right) f_{\lambda}\left(\mathbf{k}_{\mathbf{1}}, T\right)\left(1-f_{\lambda_{1}}\left(\mathbf{k}_{\mathbf{2}}, T\right)\right)\left(1-f_{\lambda_{2}}\left(\mathbf{k}_{\mathbf{3}}, T\right)\right) \\
- & V\left(\mathbf{k}-\mathbf{k}_{\mathbf{2}}\right) V\left(\mathbf{k}-\mathbf{k}_{\mathbf{3}}\right)(2 \pi)^{3} \delta\left(\omega_{1}-\epsilon_{\lambda}\left(\mathbf{k}_{\mathbf{1}}\right)\right) \delta\left(\omega_{2}-\epsilon_{\lambda_{1}}\left(\mathbf{k}_{\mathbf{2}}\right)\right) \delta\left(\omega_{3}-\epsilon_{\lambda_{2}}\left(\mathbf{k}_{\mathbf{3}}\right)\right) \\
\times & \left.M_{\lambda_{\lambda_{2}}}\left(\mathbf{k}_{\mathbf{1}}, \mathbf{k}_{\mathbf{3}}\right) M_{\lambda_{1} \lambda}\left(\mathbf{k}_{\mathbf{2}}, \mathbf{k}_{\mathbf{1}}\right) M_{\mu \lambda_{1}}\left(\mathbf{k}, \mathbf{k}_{\mathbf{2}}\right) T_{\lambda_{2} \mu}\left(\mathbf{k}_{\mathbf{3}}, \mathbf{k}\right) f_{\lambda}\left(\mathbf{k}_{\mathbf{1}}, T\right)\left(1-f_{\lambda_{1}}\left(\mathbf{k}_{\mathbf{2}}, T\right)\right)\left(1-f_{\lambda_{2}}\left(\mathbf{k}_{\mathbf{3}}, T\right)\right)\right]
\end{aligned}
$$

and

$$
\begin{aligned}
& \sigma_{\mu \mu}^{<}\left(\mathbf{k}, \omega=\epsilon_{\mu}(\mathbf{k}) ; \mathbf{R}, T\right)=\int \frac{d^{2} \mathbf{k}_{\mathbf{1}}}{(2 \pi)^{2}} \frac{d \omega_{1}}{2 \pi} \frac{d^{2} \mathbf{k}_{\mathbf{2}}}{(2 \pi)^{2}} \frac{d \omega_{2}}{2 \pi} \frac{d^{2} \mathbf{k}_{\mathbf{3}}}{(2 \pi)^{2}} \frac{d \omega_{3}}{2 \pi}(2 \pi)^{3} \delta\left(\mathbf{k}+\mathbf{k}_{\mathbf{1}}-\mathbf{k}_{\mathbf{2}}-\mathbf{k}_{\mathbf{3}}\right) \\
\times & \delta\left(\epsilon_{\mu}(\mathbf{k})+\omega_{1}-\omega_{2}-\omega_{3}\right) \times \\
\times & {\left[N V\left(\mathbf{k}-\mathbf{k}_{\mathbf{2}}\right) V\left(\mathbf{k}_{\mathbf{1}}-\mathbf{k}_{\mathbf{2}}\right)(2 \pi)^{3} \delta\left(\omega_{1}-\epsilon_{\lambda}\left(\mathbf{k}_{\mathbf{1}}\right)\right) \delta\left(\omega_{2}-\epsilon_{\lambda_{1}}\left(\mathbf{k}_{\mathbf{2}}\right)\right) \delta\left(\omega_{3}-\epsilon_{\lambda_{2}}\left(\mathbf{k}_{\mathbf{3}}\right)\right)\right.} \\
\times & M_{\lambda_{2} \lambda}\left(\mathbf{k}_{\mathbf{3}}, \mathbf{k}_{\mathbf{1}}\right) M_{\lambda_{2}}\left(\mathbf{k}_{\mathbf{1}}, \mathbf{k}_{\mathbf{3}}\right) M_{\mu \lambda_{1}}\left(\mathbf{k}, \mathbf{k}_{\mathbf{2}}\right) M_{\lambda_{1} \mu}\left(\mathbf{k}_{\mathbf{2}}, \mathbf{k}\right)\left(1-f_{\lambda}\left(\mathbf{k}_{\mathbf{1}}, T\right)\right) f_{\lambda_{1}}\left(\mathbf{k}_{\mathbf{2}}, T\right) f_{\lambda_{2}}\left(\mathbf{k}_{\mathbf{3}}, T\right) \\
- & V\left(\mathbf{k}-\mathbf{k}_{\mathbf{2}}\right) V\left(\mathbf{k}-\mathbf{k}_{\mathbf{3}}\right)(2 \pi)^{3} \delta\left(\omega_{1}-\epsilon_{\lambda}\left(\mathbf{k}_{\mathbf{1}}\right)\right) \delta\left(\omega_{2}-\epsilon_{\lambda_{1}}\left(\mathbf{k}_{\mathbf{2}}\right)\right) \delta\left(\omega_{3}-\epsilon_{\lambda_{2}}\left(\mathbf{k}_{\mathbf{3}}\right)\right) \\
\times & \left.M_{\lambda_{\lambda_{2}}}\left(\mathbf{k}_{\mathbf{1}}, \mathbf{k}_{\mathbf{3}}\right) M_{\lambda_{1} \lambda}\left(\mathbf{k}_{\mathbf{2}}, \mathbf{k}_{\mathbf{1}}\right) M_{\mu \lambda_{1}}\left(\mathbf{k}, \mathbf{k}_{\mathbf{2}}\right) M_{\lambda_{2} \mu}\left(\mathbf{k}_{\mathbf{3}}, \mathbf{k}\right)\left(1-f_{\lambda}\left(\mathbf{k}_{\mathbf{1}}, T\right)\right) f_{\lambda_{1}}\left(\mathbf{k}_{\mathbf{2}}, T\right) f_{\lambda_{2}}\left(\mathbf{k}_{\mathbf{3}}, T\right)\right],
\end{aligned}
$$

where the shorthand notation

$$
M_{\lambda \lambda_{1}}\left(\mathbf{k}, \mathbf{k}_{\mathbf{1}}\right)=\left[U_{\mathbf{k}} U_{\mathbf{k}_{1}}^{-1}\right]_{\lambda \lambda_{1}}=\frac{1}{2}\left(\begin{array}{ll}
1+\frac{K^{\star} K_{1}}{k k_{1}} & 1-\frac{K^{\star} K_{1}}{k k_{1}} \\
1-\frac{K^{\star} K_{1}}{k k_{1}} & 1+\frac{K^{\star} K_{1}}{k k_{1}}
\end{array}\right)_{\lambda \lambda_{1}}=\frac{1}{2}\left(1+\lambda \lambda_{1} \frac{K^{\star} K_{1}}{k k_{1}}\right)
$$


was introduced. The connection with the matrix elements defined in Eq. (3.9) can be easily established and reads

$$
T_{\lambda \lambda_{1} \lambda_{2} \lambda_{3}}\left(\mathbf{k}, \mathbf{k}_{\mathbf{1}}, \mathbf{q}\right)=\frac{1}{2} V(-\mathbf{q}) M_{\lambda \lambda_{3}}(\mathbf{k}+\mathbf{q}, \mathbf{k}) M_{\lambda_{1} \lambda_{2}}\left(\mathbf{k}_{\mathbf{1}}-\mathbf{q}, \mathbf{k}_{\mathbf{1}}\right) .
$$

Using Eq. (A7) and preforming a sequence of transformations finally yields

$$
\begin{aligned}
& {\left[\partial_{T}-\nabla_{\mathbf{R}} U(\mathbf{R}, T) \nabla_{\mathbf{k}}\right] f_{\mu}(\mathbf{k}, T)=\frac{2 \pi}{v_{F}} \int \frac{d^{2} \mathbf{k}_{\mathbf{1}}}{(2 \pi)^{2}} \frac{d^{2} \mathbf{q}}{(2 \pi)^{2}} \delta\left(\mu k+\lambda k_{1}-\lambda_{1}|\mathbf{k}+\mathbf{q}|-\lambda_{2}\left|\mathbf{k}_{\mathbf{1}}-\mathbf{q}\right|\right) } \\
\times & {\left[N V(-\mathbf{q}) V(-\mathbf{q}) M_{\lambda_{2} \lambda}\left(\mathbf{k}_{\mathbf{1}}-\mathbf{q}, \mathbf{k}_{\mathbf{1}}\right) M_{\lambda \lambda_{2}}\left(\mathbf{k}_{\mathbf{1}}, \mathbf{k}_{\mathbf{1}}-\mathbf{q}\right) M_{\mu \lambda_{1}}(\mathbf{k}, \mathbf{q}+\mathbf{k}) M_{\lambda_{1} \mu}(\mathbf{q}+\mathbf{k}, \mathbf{k})\right.} \\
- & \left.V(-\mathbf{q}) V\left(\mathbf{k}-\mathbf{k}_{\mathbf{1}}+\mathbf{q}\right) M_{\lambda \lambda_{2}}\left(\mathbf{k}_{\mathbf{1}}, \mathbf{k}_{\mathbf{1}}-\mathbf{q}\right) M_{\lambda_{1} \lambda}\left(\mathbf{q}+\mathbf{k}, \mathbf{k}_{\mathbf{1}}\right) M_{\mu \lambda_{1}}(\mathbf{k}, \mathbf{q}+\mathbf{k}) M_{\lambda_{2} \mu}\left(\mathbf{k}_{\mathbf{1}}-\mathbf{q}, \mathbf{k}\right)\right] \\
& {\left[\left(1-f_{\mu}(\mathbf{k}, T)\right)\left(1-f_{\lambda}\left(\mathbf{k}_{\mathbf{1}}, T\right)\right) f_{\lambda_{1}}(\mathbf{q}+\mathbf{k}, T) f_{\lambda_{2}}\left(\mathbf{k}_{\mathbf{1}}-\mathbf{q}, T\right)\right.} \\
- & \left.f_{\mu}(\mathbf{k}, T) f_{\lambda}\left(\mathbf{k}_{\mathbf{1}}, T\right)\left(1-f_{\lambda_{1}}(\mathbf{q}+\mathbf{k}, T)\right)\left(1-f_{\lambda_{2}}\left(\mathbf{k}_{\mathbf{1}}-\mathbf{q}, T\right)\right)\right] .
\end{aligned}
$$

In a next step we will make connection to the Golden rule result of the main text. It is straightforward to see that using Eq. (A10) we can rewrite the above expression to yield

$$
\begin{aligned}
& {\left[\partial_{T}-\nabla_{\mathbf{R}} U(\mathbf{R}, T) \nabla_{\mathbf{k}}\right] f_{\mu}(\mathbf{k}, T)=\frac{2 \pi}{v_{F}} \int \frac{d^{2} \mathbf{k}_{\mathbf{1}}}{(2 \pi)^{2}} \frac{d^{2} \mathbf{q}}{(2 \pi)^{2}} \delta\left(\mu k+\lambda k_{1}-\lambda_{1}|\mathbf{k}+\mathbf{q}|-\lambda_{2}\left|\mathbf{k}_{\mathbf{1}}-\mathbf{q}\right|\right) } \\
\times & 4\left[N\left|T_{\mu \lambda \lambda_{2} \lambda_{1}}\left(\mathbf{k}, \mathbf{k}_{\mathbf{1}}, \mathbf{q}\right)\right|^{2}-T_{\mu \lambda \lambda_{2} \lambda_{1}}\left(\mathbf{k}, \mathbf{k}_{\mathbf{1}}, \mathbf{q}\right) T_{\mu \lambda \lambda_{1} \lambda_{2}}^{\star}\left(\mathbf{k}, \mathbf{k}_{\mathbf{1}}, \mathbf{k}_{\mathbf{1}}-\mathbf{k}-\mathbf{q}\right)\right] \\
& {\left[\left(1-f_{\mu}(\mathbf{k}, T)\right)\left(1-f_{\lambda}\left(\mathbf{k}_{\mathbf{1}}, T\right)\right) f_{\lambda_{1}}(\mathbf{q}+\mathbf{k}, T) f_{\lambda_{2}}\left(\mathbf{k}_{\mathbf{1}}-\mathbf{q}, T\right)\right.} \\
- & \left.f_{\mu}(\mathbf{k}, T) f_{\lambda}\left(\mathbf{k}_{\mathbf{1}}, T\right)\left(1-f_{\lambda_{1}}(\mathbf{q}+\mathbf{k}, T)\right)\left(1-f_{\lambda_{2}}\left(\mathbf{k}_{\mathbf{1}}-\mathbf{q}, T\right)\right)\right]
\end{aligned}
$$

Energy and momentum conservation restricts the valid combinations of particles and holes scattering, see Ref. [11, 13], which simplifies the above expression. Applying all these simplifications and shifting the variables appropriately we obtain

$$
\begin{gathered}
{\left[\partial_{T}-\nabla_{\mathbf{R}} U(\mathbf{R}, T) \nabla_{\mathbf{k}}\right] f_{\mu}(\mathbf{k}, T)=-\frac{(2 \pi)}{v_{F}} \int \frac{d^{2} k_{1}}{(2 \pi)^{2}} \frac{d^{2} k_{2}}{(2 \pi)^{2}}\{} \\
\delta\left(k-k_{1}-|\mathbf{k}+\mathbf{q}|+\left|\mathbf{k}_{1}-\mathbf{q}\right|\right) \bar{R}_{1}\left\{f_{\mu}(\mathbf{k}, t) f_{-\mu}\left(\mathbf{k}_{1}, t\right)\left[1-f_{\mu}(\mathbf{k}+\mathbf{q}, t)\right]\left[1-f_{-\mu}\left(\mathbf{k}_{1}-\mathbf{q}, t\right)\right]\right. \\
\left.-\left[1-f_{\mu}(\mathbf{k}, t)\right]\left[1-f_{-\mu}\left(\mathbf{k}_{1}, t\right)\right] f_{\mu}(\mathbf{k}+\mathbf{q}, t) f_{-\mu}\left(\mathbf{k}_{1}-\mathbf{q}, t\right)\right\} \\
\delta\left(k+k_{1}-|\mathbf{k}+\mathbf{q}|-\left|\mathbf{k}_{1}-\mathbf{q}\right|\right) \bar{R}_{2}\left\{f_{\mu}(\mathbf{k}, t) f_{\mu}\left(\mathbf{k}_{1}, t\right)\left[1-f_{\mu}(\mathbf{k}+\mathbf{q}, t)\right]\left[1-f_{\mu}\left(\mathbf{k}_{1}-\mathbf{q}, t\right)\right]\right. \\
\left.\left.-\left[1-f_{\mu}(\mathbf{k}, t)\right]\left[1-f_{\mu}\left(\mathbf{k}_{1}, t\right)\right] f_{\mu}(\mathbf{k}+\mathbf{q}, t) f_{\mu}\left(\mathbf{k}_{1}-\mathbf{q}, t\right)\right\}\right\}, \quad(\mathrm{A} 13)
\end{gathered}
$$

where

$$
\begin{aligned}
\bar{R}_{1}= & 4 N\left(\left|T_{+--+}\left(\mathbf{k}, \mathbf{k}_{\mathbf{1}}, \mathbf{q}\right)\right|^{2}+\left|T_{+-+-}\left(\mathbf{k}, \mathbf{k}_{\mathbf{1}}, \mathbf{k}_{\mathbf{1}}-\mathbf{k}-\mathbf{q}\right)\right|^{2}\right) \\
& -4 T_{+--+}\left(\mathbf{k}, \mathbf{k}_{\mathbf{1}}, \mathbf{q}\right) T_{+-+-}^{\star}\left(\mathbf{k}, \mathbf{k}_{\mathbf{1}}, \mathbf{k}_{\mathbf{1}}-\mathbf{k}-\mathbf{q}\right) \\
& -4 T_{+-+-}^{\star}\left(\mathbf{k}, \mathbf{k}_{\mathbf{1}}, \mathbf{k}_{\mathbf{1}}-\mathbf{k}-\mathbf{q}\right) T_{+--+}\left(\mathbf{k}, \mathbf{k}_{\mathbf{1}}, \mathbf{q}\right)
\end{aligned}
$$


and

$$
\begin{aligned}
\bar{R}_{2}= & 4 N\left|T_{++++}\left(\mathbf{k}, \mathbf{k}_{\mathbf{1}}, \mathbf{q}\right)\right|^{2} \\
& -4 T_{++++}\left(\mathbf{k}, \mathbf{k}_{\mathbf{1}}, \mathbf{q}\right) T_{++++}^{\star}\left(\mathbf{k}, \mathbf{k}_{\mathbf{1}}, \mathbf{k}_{\mathbf{1}}-\mathbf{k}-\mathbf{q}\right) .
\end{aligned}
$$

Performing the appropriate shifts allows to write

$$
\begin{aligned}
\bar{R}_{1}= & 4(N-1)\left|T_{+--+}\left(\mathbf{k}, \mathbf{k}_{\mathbf{1}}, \mathbf{q}\right)\right|^{2}+4(N-1)\left|T_{+-+-}\left(\mathbf{k}, \mathbf{k}_{\mathbf{1}}, \mathbf{k}_{\mathbf{1}}-\mathbf{k}-\mathbf{q}\right)\right|^{2} \\
& +4\left|T_{+--+}\left(\mathbf{k}, \mathbf{k}_{\mathbf{1}}, \mathbf{q}\right)-T_{+-+-}\left(\mathbf{k}, \mathbf{k}_{\mathbf{1}}, \mathbf{k}_{\mathbf{1}}-\mathbf{k}-\mathbf{q}\right)\right|^{2}
\end{aligned}
$$

and

$$
\begin{aligned}
\bar{R}_{2}= & 4(N-1)\left|T_{++++}\left(\mathbf{k}, \mathbf{k}_{\mathbf{1}}, \mathbf{q}\right)\right|^{2} \\
& +2\left|T_{++++}\left(\mathbf{k}, \mathbf{k}_{\mathbf{1}}, \mathbf{q}\right)-T_{++++}\left(\mathbf{k}, \mathbf{k}_{\mathbf{1}}, \mathbf{k}_{\mathbf{1}}-\mathbf{k}-\mathbf{q}\right)\right|^{2},
\end{aligned}
$$

which establishes the equivalence of Fermi's Golden rule and the Keldysh treatment, see Eq. (3.23).

[1] A. H. Castro Neto, F. Guinea, N. M. R. Peres, K. S. Novoselov, and A. K. Geim, arXiv:0709:1163.

[2] K. I. Bolotin, K. J. Sikes, Z. Jiang, G. Fudenberg, J. Hone, P. Kim, and H. L. Stormer, arXiv:0802.2389.

[3] X. Du, I. Skachko, A. Barker, and E. Y. Andrei, arXiv:0802.2933.

[4] M. Müller and S. Sachdev, arXiv:0801.2970

[5] D. T. Son, Phys. Rev. B 75, 235423 (2007).

[6] J. González, F. Guinea, and V. A. M. Vozmediano, Nucl. Phys. B 424, 595 (1994); Phys. Rev. B 59, R2474 (1999).

[7] J. Ye and S. Sachdev, Phys. Rev. Lett. 80, 5409 (1998).

[8] E. V. Gorbar, V. P. Gusynin, V. A. Miransky, and I. A. Shovkovy, Phys. Rev. B 66, 045108 (2002).

[9] D. E. Sheehy and J. Schmalian, Phys. Rev. Lett. 99, 226803 (2007).

[10] K. Damle and S. Sachdev, Phys. Rev. B 56, 8714 (1997).

[11] S. Sachdev, Phys. Rev. B 57, 7157 (1998).

[12] I. F. Herbut, V. Juričić, and O. Vafek, Phys. Rev. Lett. 100, 046403 (2008).

[13] S. Sachdev, Quantum Phase Transitions, Cambridge University Press, Cambridge (1999), pp. 185.

[14] A. Kashuba, arXiv:0802.2216.

[15] P. Kovtun and L. G. Yaffe, Phys. Rev. D 68, 025007 (2003).

[16] I. S. Terekhov, A. I. Milstein, V. N. Kotov, and O. P. Sushkov, Phys. Rev. Lett. 100, 076803 (2008). 
[17] R. R. Biswas, S. Sachdev, and D. T. Son, Phys. Rev. B 76, 205122 (2007).

[18] P. Arnold, G. D. Moore and L. G. Yaffe, JHEP 11, 001 (2000).

[19] J. M. Ziman, Electrons and Phonons, Oxford University Press, Oxford (1960), Chapter 7.

[20] M. S. Foster and I. L. Aleiner, arXiv:0802.0283.

[21] L. P. Kadanoff and G. Baym, Quantum Statistical Mechanics (Benjamin, New York, 1962) . 